\title{
Ultrafast X-ray Absorption Studies of the Structural Dynamics of Molecular and Biological Systems in Solution
}

\author{
Christopher J. Milne, Renske M. Van der Veen, Van-Thai Pham, Frederico A. Lima, Hannelore \\ Rittmann-Frank, Marco Reinhard, Frank van Mourik, Susanne Karlsson, Thomas J. Penfold, \\ and Majed Chergui*
}

\begin{abstract}
We review our recent studies of excited state structures and dynamics of chemical and biological systems with pico- and femtosecond X-ray absorption spectroscopy in the liquid phase.
\end{abstract}

Keywords: X-ray absorption spectroscopy

\section{Introduction}

Chemical reactivity and biological function are intimately linked to structure and structural dynamics on the molecular level. The advent of ultrafast spectroscopy in the mid-1980s has allowed the probing of chemical reactions in real-time. ${ }^{[1]}$ However, these studies are carried out using probe pulses in the optical domain, which only delivers information on the electronic structure. Therefore, over the past 10-15 years a huge effort was made aimed at using electron or X-ray probe pulses that can deliver geometric structural information such as bond lengths and bond angles, via scattering, diffraction, or X-ray spectroscopy which additionally delivers information about the electronic structure. ${ }^{[2]}$ Our primary efforts have mostly been focussed on developing and using time-domain $\mathrm{X}$ ray absorption spectroscopy (XAS). ${ }^{[3,4]} \mathrm{X}$ ray absorption spectra are characterized by absorption edges, which are due to excitation of the core electrons of a given atom to the ionization threshold. An absorption edge consists of two regions: the X-ray absorption near edge structure (XANES) at

\footnotetext{
${ }^{*}$ Correspondence: Prof. Dr. M. Chergui

École Polytechnique Fédérale de Lausanne (EPFL) Laboratory of Ultrafast Spectroscopy

EPFL-SB-ISIC-LSU

Station 6

$\mathrm{CH}-1015$ Lausanne

Tel.: + 41216930447

Fax: + 41216930365

E-mail: majed.chergui@epfl.ch
}

energy just below and just above (typically $<50 \mathrm{eV}$ ) the edge and the extended X-ray absorption fine structure (EXAFS), which lies at higher energies (typically $>50 \mathrm{eV}$ ) above the edge. For an atom embedded in a molecule, crystal or even a liquid, both XANES and EXAFS exhibit modulations, which are due to the multiple and single scattering of the X-ray excited photoelectron onto the nearest neighbours.

Our choice of XAS is motivated by the fact that: a) it is element specific; b) it delivers information about the local structure (coordination numbers and bond distances) around the atom of interest, c) just below the edge bound-bound transitions occur, which contain information about the electronic structure (such as oxidation state, occupancy of valence orbitals, charge transfer, etc.) of the system; d) last but not least, it can be implemented in any phase of matter, and in particular in liquids, which are the natural environment of biology and of most of chemistry.

Time-resolved XAS requires tunable $\mathrm{X}$-radiation with sufficiently short pulses, which can be synchronized to an external light source (e.g. a pump laser). Storage rings, such as Swiss Light Source (SLS) at the Paul-Scherrer-Institut in Villigen, offer the required specificities in terms of flux, energy range and stability, but the synchrotron X-ray pulses have widths of 50-100 ps. Therefore, in order to push into the femtosecond time domain, which is the fundamental time scale in chemistry, materials science and biology, the slicing scheme was implemented in the hard Xray range at the MicroXas beamline of the SLS. It provides 100 fs hard X-ray pulses albeit at the cost of a dramatic reduction in flux. ${ }^{[5]}$ In all cases (ps or fs) the methodology is based on recording the transient (difference spectra of the excited minus the unexcited sample) absorption on a pulse- to-pulse basis. ${ }^{[6]}$ In the following sections, we illustrate the methodology on a few examples and how it helped answer specific scientific questions.

\section{Intramolecular Charge Transfer in Metal Complexes}

Transition metal complexes play an important role in chemistry and in many cases in biology. They are also of importance for solar energy research and for the development of molecular devices. Their visible absorption spectrum is dominated by the metal-to-ligand-charge-transfer (MLCT) bands. For the MLCT states, there is an issue of structural changes as a result of charge transfer. We addressed this question in a picosecond XAS study of aqueous ruthenium(II)-tris-2,2'-bipyridine, $\left(\left[\mathrm{Ru}^{\mathrm{II}}(\mathrm{bpy})_{3}\right]^{2+}\right) \cdot{ }^{[7]}$ The system was excited in the ${ }^{1}$ MLCT band, which leads to population of the ${ }^{3} \mathrm{MLCT}$ state on ultrafast time scales. ${ }^{[8]}$ The $\mathrm{L}_{3}\left(2 \mathrm{p}_{3 / 2}-4 \mathrm{~d}\right)$ and $\mathrm{L}_{2}$ $\left(2 \mathrm{p}_{1 / 2}-4 \mathrm{~d}\right)$ edges of ruthenium were probed, delivering information on the electronic structure, i.e. the occupancy of the $\mathrm{d}\left(\mathrm{t}_{2 \mathrm{~g}}\right)$ and $d\left(e_{g}\right)$ orbitals in the excited triplet state. Information about the excited state structure was obtained via the electronic structure (based on the relationship between ligand field splitting and coordinating structure) and via the EXAFS at the $\mathrm{L}_{3}$ edge. Both showed that the structure change is a small $(-0.037 \pm 0.0135 \AA)$ contraction of the $\mathrm{Ru}-\mathrm{N}$ bonds. ${ }^{[7 \mathrm{~b}]}$ This relatively weak bond contraction is due to a balance between strong attractive forces between metal and ligand after charge transfer, and steric effects resulting from the three bpy ligands being in a constrained geometry, already in the ground state. Our results were later confirmed by quantum chemical calculations. ${ }^{[9]}$ 
Similar studies have also been carried out on halogenated rhenium-carbonyl polypyridine complexes by probing the electronic and geometric structure at both the L-edges of rhenium and the K-edge of the halogen ligand. These will be discussed in a forthcoming publication.

\section{Bond Formation in Bimetallic Complexes}

The triplet excited states of binuclear $\mathrm{d}^{8}-\mathrm{d}^{8}$ platinum, rhodium, and iridium complexes (bridged by various ligands) have attracted much attention owing to their remarkable photophysical and photochemical properties, which are strongly determined by their structure. We investigated the tetrakis- $\mu$-pyrophosphitodiplatinate(II) $\left[\mathrm{Pt}_{2}\left(\mathrm{P}_{2} \mathrm{O}_{5} \mathrm{H}_{2}\right)_{4}\right]^{4-}$ ion, which is the most studied binuclear metal complex due, among other reasons, to its photocatalytic activity. In the ground state the two metal atoms are not chemically bound, but excitation of the first singlet state results in a bond owing to the promotion of an electron from the antibonding $d \sigma^{*}\left(\mathrm{~d}_{\mathrm{z}}{ }^{2}\right.$-derived $)$ to the bonding $p \sigma$ ( $\mathrm{p}_{\mathrm{z}}$-derived) orbitals, which leads to a contraction of the metal-metal bond. The system then undergoes intersystem crossing to the long lived ( $1 \mu \mathrm{s})$ lowest triplet states.[10] Using time-resolved XAS, van der Veen et al. resolved its geometric $^{[11]}$ and electronic ${ }^{[12]}$ structure via EXAFS and XANES, respectively. Fig. 1 shows the ground state $\mathrm{Pt} \mathrm{L}_{3}$-edge XAS spectrum (black trace) as well as the transient spectrum, integrated from 0 to 150 ns to improve the signal-to-noise ratio. The inset shows the XANES region for the ground-state complex and its transient spectrum, wherein dramatic changes appear. In particular, a new absorption shows up at $11.574 \mathrm{keV}$, just below the absorption edge, which is due to the creation of a hole in the $5 \mathrm{~d} \sigma^{*}$ orbital upon laser excitation that can then be accessed from the $2 \mathrm{p}_{3 / 2}$ core orbital $\left(\mathrm{L}_{3}\right.$ edge $) .{ }^{[12]}$ Clear changes are visible in the EXAFS region (Fig. 1b), reflecting structural modifications between the ground and excited triplet states.

From the transient EXAFS spectrum (Fig. 1b), the magnitude of the Pt-Pt bond contraction as well as, for the first time, the changes affecting the ligand bonds were extracted. In particular (see scheme in Fig. 1b), while the Pt-Pt bond contracts, the $\mathrm{Pt}-\mathrm{P}$ bonds slightly elongate, in agreement with theoretical predictions. ${ }^{[13]}$ This work underscores the ability to retrieve details of the excited state structure of a rather complex molecular system in solution. However, the X-ray determined structure deviates somewhat from that obtained from optical spectra (the system happens to be highly harmonic in both the ground and the ex-



Fig. 1. a) Static Pt $\mathrm{L}_{3}$ XAS spectrum of $\left[\mathrm{Pt}_{2}\left(\mathrm{P}_{2} \mathrm{O}_{5} \mathrm{H}_{2}\right)_{4}\right]^{4-}$ in solution (black line, left axis) and the transient (excited - unexcited) XAS spectrum (red circles, right axis) integrated up to $150 \mathrm{~ns}$ after excitation. The inset zooms into the XANES region. b) Transient EXAFS data and best fit (solid line) with the following results: a Pt-Pt contraction of 0.31(5) Å, a Pt-ligand bond elongation of $0.010(6) \AA{ }^{[} \cdot{ }^{[1]}$

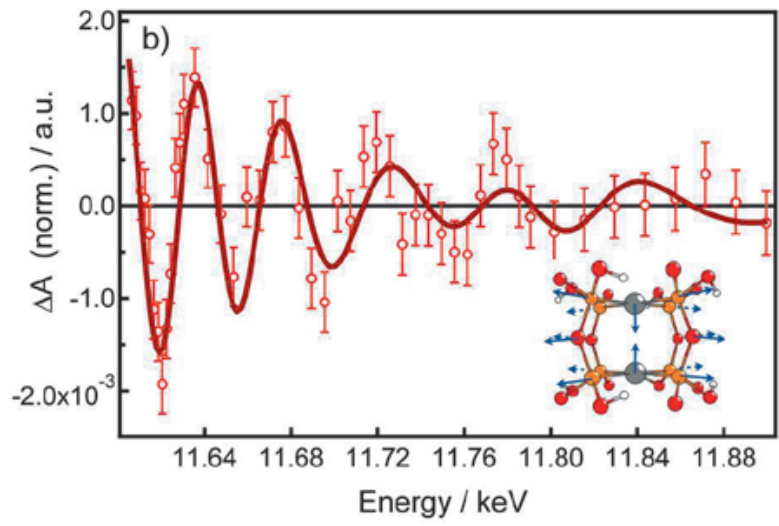

cited state), ${ }^{[10]}$ and we believe that this may stem from the neglect of the solvent contribution in modeling the EXAFS. These effects and eventually coordination of solvent molecules to the excited complex is an aspect which we are now investigating in detail (see below).

\section{Spin Cross-over Complexes}

One of the fascinating features of $\mathrm{Fe}$ (II)-polypyridine complexes is their ability to undergo dramatic spin changes under temperature, pressure or irradiation. They have therefore been named spin crossover complexes (SCO complexes). ${ }^{[14]}$ In the predominantly octahedral field of the ligands, all electrons are in the lower $t_{2 \mathrm{~g}}$ sub-shell forming the low spin (LS) ground state, while transferring electrons to the $e_{\mathrm{g}}$ orbitals increases the spin state. Because the $\mathrm{e}_{\mathrm{g}}$ orbitals derive from the $\mathrm{d}_{\mathrm{x}-\mathrm{y}}^{2} 2$ and $\mathrm{d}_{\mathrm{z}}^{2}$ orbitals, they are antibonding in six-fold coordinated complexes, which leads to a striking metal-ligand bond elongation in the high spin (HS) state. A generic diagram of the potential energy curves of $\mathrm{Fe}$ (II) SCO complexes is shown in the inset to Fig. 2, as a function of the $\mathrm{Fe}-\mathrm{N}$ bond length. The ligand field states ${ }^{1,3} \mathrm{~T},{ }^{5} \mathrm{~T}$ and ${ }^{5} \mathrm{E}$ have their equilib- rium distances elongated by $\sim 0.1 \AA, \sim 0.2$ $\AA$, and $\sim 0.3 \AA$, respectively, relative to the ground state (and MLCT) bond distance. Light excitation into the ${ }^{1}$ MLCT state leads to population of the lowest quintet state ${ }^{5} \mathrm{~T}_{2}$ with unity quantum yield. ${ }^{[14]}$ For the $\left.\left[\mathrm{Fe}^{2} \text { (bpy }\right)_{3}\right]^{2+}$ complex, which is the smallest of the family of $\mathrm{Fe}(\mathrm{II})$-complexes, the quintet state lifetime is the shortest, $\sim 650$ ps at room temperature. As a consequence, the quintet state structure could not be determined by quasi-static methods (X-ray diffraction or X-ray absorption spectroscopy). One of the questions that arises is whether the quintet lifetime is determined by the $\mathrm{Fe}$-ligand bond distance, bearing in mind that nearly all SCO complexes exhibit the same bond elongation (typically $\sim 0.2 \AA$ ) in the HS state. ${ }^{[17]}$ We determined its HS state structure by recording the $\mathrm{Fe} \mathrm{K}$ edge XAS of the system with 70 ps X-ray pulses. ${ }^{[18]}$ Fig. 3 shows the XANES of the molecule in the ground state, the transient (difference) spectrum at 50 ps time delay and the XAS spectrum of the quintet state, as retrieved from the ground state and the difference spectra and from the photolysis yield determined in laser-only experiments (and convoluted to match the much longer X-ray probe width). The structural analysis of the excited state delivered an $\mathrm{Fe}-\mathrm{N}$ bond elongation of $0.203 \pm 0.008$ 


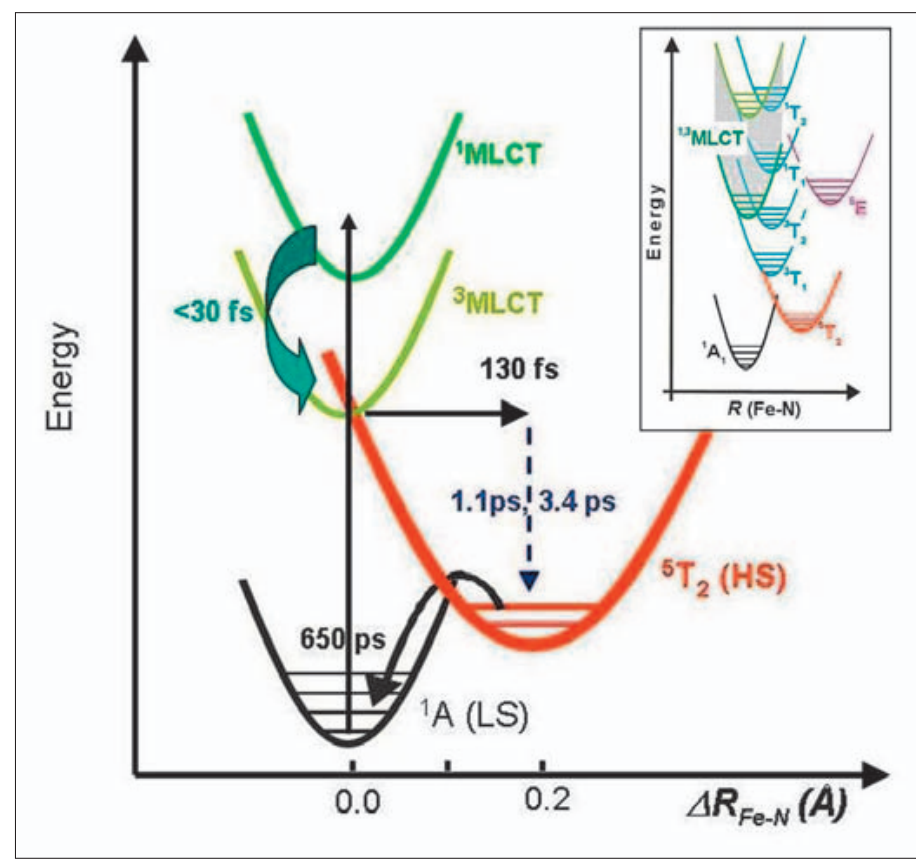

Fig. 2. Photocycle of the $\left[\mathrm{Fe}^{\prime \prime}(\mathrm{bpy})_{3}\right]^{2+}$ complex in aqueous solution as determined from our ultrafast optical and X-ray spectroscopy studies. ${ }^{[15]}$ The inset shows typical potential energy curves of $\mathrm{Fe}$ (II) polypyridine complexes as a function of the $\mathrm{Fe}-\mathrm{N}$ bond distance adapted from ref. [16]. The manifold of metal-to-ligand-charge-transfer (MLCT) states is shown as a shaded area. The metal-centred (MC) states are represented by their symmetry character ( $A, T$ and $E$ ) in the $D_{3}$ group.

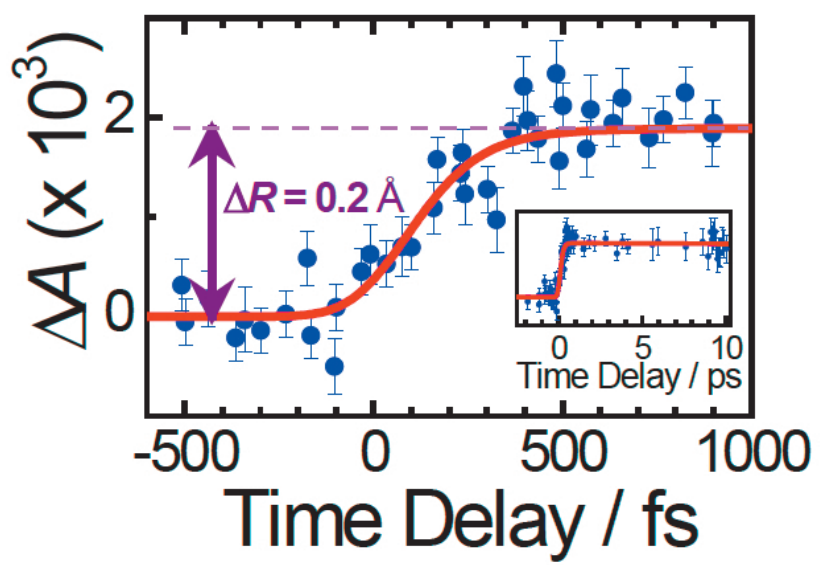

Fig. 4. Femtosecond XANES results of aqueous $\left[\mathrm{Fe} \mathrm{e}^{\prime \prime}(\mathrm{bpy})_{3}\right]^{2+:}$ Time scan of the signal (blue points) at the B-feature (Fig. 3), as a function of laser pump/X-ray probe time delay after $400 \mathrm{~nm}$ excitation. The inset shows a long-time scan up to $10 \mathrm{ps}$ time delay. The red trace is the simulated signal assuming a rise time of $\sim 150 \mathrm{fs}$. The transient absorption signal is directly proportional to the $\mathrm{Fe}-\mathrm{N}$ bond elongation and its value beyond 300 fs corresponds to that of the quintet state.

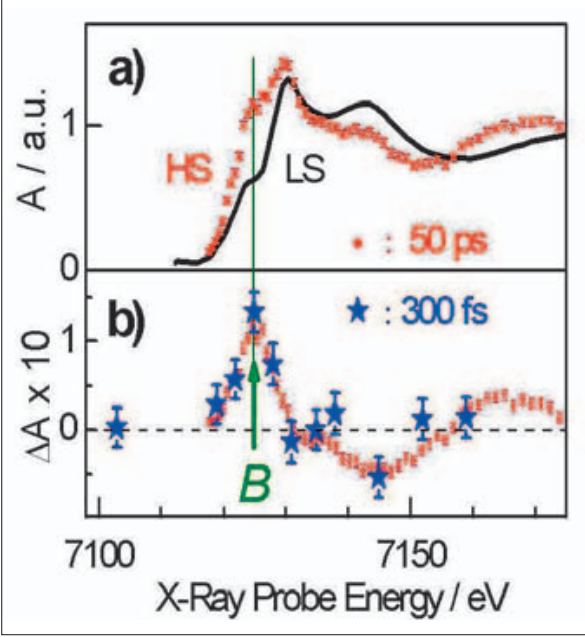

Fig. 3. a) static ground state XANES spectrum of ground state aqueous $\left[\mathrm{Fe}^{\prime \prime}(\mathrm{bpy})_{3}\right]^{2+}$ (black trace) and in the excited high spin quintet state (red points), as obtained from the transient spectrum at 50 ps shown in (b) (red dots). The transient spectrum at $300 \mathrm{fs}$ time delay is shown as blue stars in (b).

A. ${ }^{119]}$ The fact that the bond elongation in the HS state is nearly the same as all other Fe(II)-complexes[17] shows that this is not the parameter that controls the HS lifetime. Rather, the adiabatic energy and the coupling parameters between LS and HS states are the crucial parameters. Indeed, of all $\mathrm{Fe}(\mathrm{II})-\mathrm{SCO}$ complexes, $\left[\mathrm{Fe}^{\mathrm{II}}(\mathrm{bpy})_{3}\right]^{2+}$ has the highest lying quintet state, and therefore the force that drives the system back to the LS state is largest in this case.

The mechanism and relaxation pathways of the above photoinduced HSLS conversion in $\mathrm{Fe}(\mathrm{II})$ complexes have long been debated.[14] It was generally admitted that the population relaxes via the intermediate ligand-field $\mathrm{T}$ states in a step-wise fashion down to the quintet state. The early time dynamics was identified by Gawelda et al. in laser-only studies, ${ }^{[20]}$ and showed that departure from the ${ }^{3}$ MLCT state occurs in $\sim 130$ fs (Fig. 2). The ground state XANES spectrum shows a multiple scattering band (the B-feature, Fig. 3) whose amplitude is nearly linear with $\mathrm{Fe}-\mathrm{N}$ bond elongation. We used this relationship to probe the arrival of the population in the quintet state by femtosecond XAS using the slicing scheme. Fig. 4 shows the time scan obtained at the B-feature $(7.122 \mathrm{keV})$. The signal stabilizes from about 300 fs up to the scan limit of $10 \mathrm{ps}$ (see inset), which is evidence that the system is already in the quintet state. Further evidence is that the energy scan at 300 fs reproduces the transient spectrum at 50 ps time resolution (Fig. 3b). The fit in Fig. 4 shows that the quintet state is reached in $150 \pm 50 \mathrm{fs}$, in agreement with the decay of the ${ }^{3}$ MLCT state, ${ }^{[20]}$ implying that the ultrafast spin conversion is a simple three-step ${ }^{1} \mathrm{MLCT}-{ }^{3} \mathrm{MLCT}-{ }^{5} \mathrm{~T}$, cascade that bypasses the intermediate ${ }^{1,3} \mathrm{~T}$ states. Further visible pump/UV probe (in the 300-340 $\mathrm{nm}$ region) transient absorp- tion experiments confirmed this conclusion and identified the vibrational relaxation dynamics in the quintet state, ${ }^{[21]}$ thus providing a complete picture of the photocycle of aqueous $\left[\mathrm{Fe}^{\mathrm{II}}(\mathrm{bpy})_{3}\right]^{2+}$, which is summarised in Fig. 2.

\section{Hydrophobicity}

XAS is an ideal tool for probing the solvent shell structure around atomic solutes. ${ }^{[22]}$ For halide ions in aqueous media, a dramatic change of solvent shell structure is expected in going from the halide to the halogen case, in what has been termed hydrophilic and hydrophobic solvation, respectively. ${ }^{[23]}$ For the case of aqueous iodide, the electron can be abstracted by UV excitation below $260 \mathrm{~nm}$ or by multi photon excitation at $400 \mathrm{~nm}$. The latter approach was used by us and the formation of the $\mathrm{I}^{0}$ atom was probed via the $\mathrm{L}_{1}(2 \mathrm{~s}$ $5 p)$ edge, which delivers electronic information, while the probing at the $\mathrm{L}_{3}$ edge delivers structural information about the solvent shell via EXAFS.[24]

We recently investigated the transition from hydrophilic to hydrophobic behaviour by femtosecond XAS and both classical and quantum mechanics/molecular mechanics (QMMM) molecular dynamics (MD) simulations. ${ }^{[25]}$ The general picture that emerges is that formation of a hydrophobic cavity takes typically 4 ps to be completed. This is captured by the QMMM 


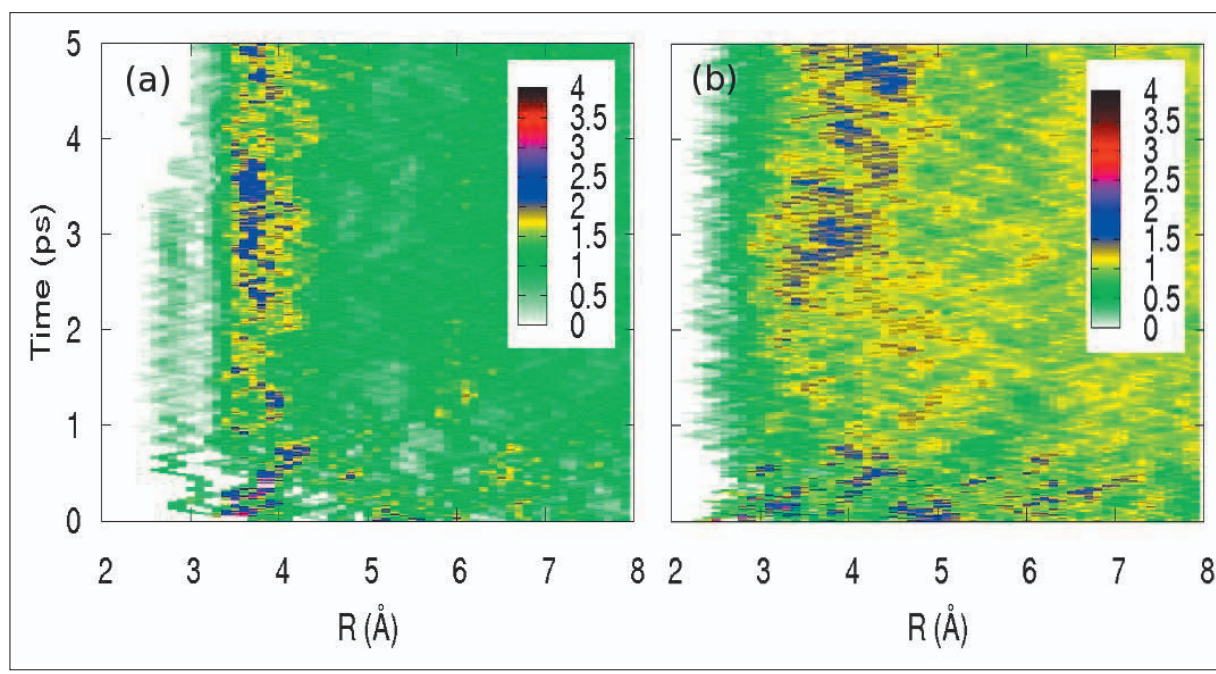

Fig. 5. a) Time evolution of the $\mathrm{I}^{0} \mathrm{-O}$ radial distribution for the first $4 \mathrm{ps}$ following the removal of an electron from iodide, obtained from an average over 10 starting configurations at each time step; b) same for $\mathrm{I}^{0} \mathrm{-H}$. The trajectories were calculated by Quantum mechanical $/$ molecular mechanics (QMMM) molecular dynamics simulations.

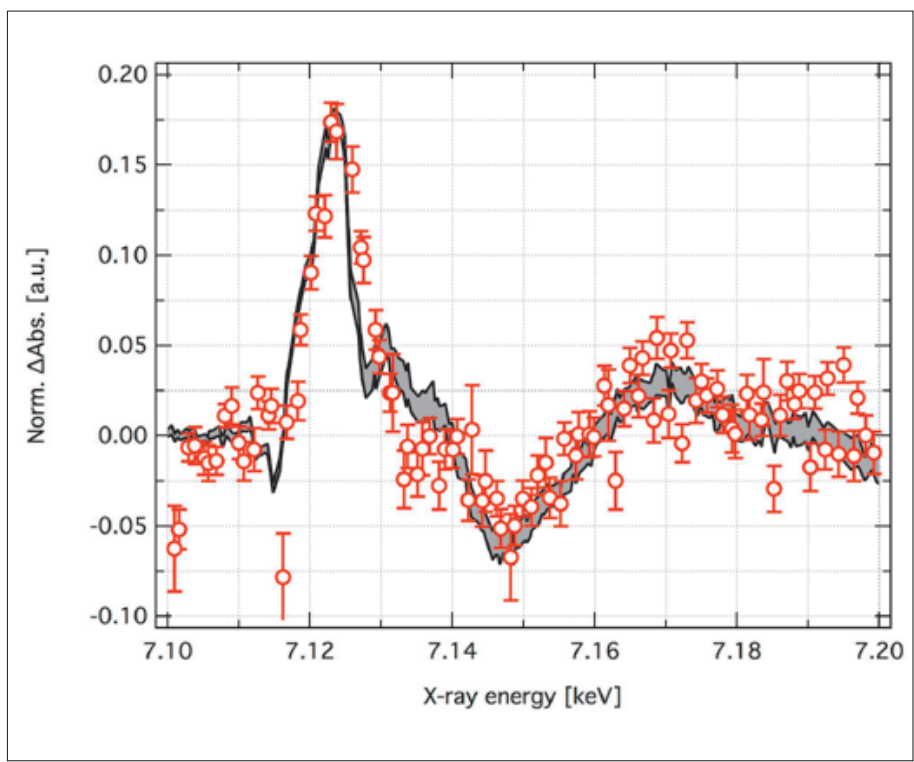

Fig. 6. Transient XAS of $4 \mathrm{mM}$ $\mathrm{MbCO}$ collected in fluorescence mode (red points with error bars). The solid trace shows the difference between the static spectra of deoxyMb and $\mathrm{MbCO}$, with the grey area representing the uncertainty of this difference.

MD simulations of the $\mathrm{I}-\mathrm{O}$ and $\mathrm{I}-\mathrm{H}$ radial distribution function as a function of time after electron abstraction (see Fig. 5). Indeed, while at short times most of the water molecules move away from the neutral solute, one molecule moves closer and stays attached to it for about 3.5 ps (Fig. 5a). During that time, the main solvent shell is reforming, with the $\mathrm{H}$-atoms reorienting to point away from the solute (contrary to the iodide case). Once this stage is completed, the bulk solvent exerts a sufficiently large force that overwhelms the van der Waalstype interaction between iodine and the single water molecule, so that the latter is pulled back to the bulk and hydrophobic solvation is complete.

\section{Biological Systems}

The above ps results were all carried out on systems of fairly high concentrations (10's of mM) using a pump laser at $1 \mathrm{kHz}$, while the synchrotron delivers $\mathrm{X}$ ray pulses at $1.04 \mathrm{MHz}$. In order to exploit this higher repetition rate, and therefore increase the $\mathrm{S} / \mathrm{N}$ ratio, we implemented a new laser set-up running at $520 \mathrm{kHz}$, and tested it on the aqueous $\left[\mathrm{Fe}^{\mathrm{II}}(\mathrm{bpy})_{3}\right]^{2+}$ sample. This set-up allows us to perform experiments at lower concentration samples, such as biological ones. As anticipated ${ }^{[6 c]}$ an increase of the $\mathrm{S} / \mathrm{N}$ by a factor of 20 was derived, which allows the study of biological samples. As a proof of principle, Fig. 6 shows the first ps pump-probe $\mathrm{Fe} \mathrm{K}$-edge transient of Myoglobin-CO (MbCO) in a physiological solution obtained at different time delays upon abstraction of the $\mathrm{CO}$ from the Fe atom. Because of the long lifetime of the system, these data were not collected on a pulse-to-pulse basis, but rather using the so-called asynchronous mode. ${ }^{[6 \mathrm{~d}]}$ Ps XAS transients have also been obtained on the MbNO system and will be discussed in a forthcoming publication.

\section{Time-resolved Soft X-ray Studies of Liquid Systems}

Extension of time-resolved XAS to the soft X-ray regime ${ }^{[3 a, 6 c]}$ offers very exciting new advantages: a) most L-edge spectra of transition metals lie in the $200-2000 \mathrm{eV}$ region; b) it allows the probing of light elements such as $\mathrm{C}, \mathrm{N}, \mathrm{O}, \mathrm{S}, \mathrm{P}$, etc. which are very important in biology. The limitation of soft X-ray spectroscopies of high vapour pressure samples has been recently overcome by the use of high speed liquid jets in vacuum chambers or of cells equipped with soft X-ray transparent windows (silicon nitride windows). ${ }^{[26]}$ Recently, time-resolved soft X-ray absorption spectroscopy have entered the picosecond time domain by probing transient changes of liquid water heated by an ultrashort infrared laser pulse, ${ }^{[27]}$ or the photoinduced changes in SCO complexes in solution in cells equipped with $\mathrm{SiN}$ windows. ${ }^{[28]}$ As a first step towards timeresolved studies of biological systems and in collaboration with the BESSY synchrotron in Berlin, we demonstrated the feasibility of soft X-ray absorption spectroscopy of biological systems in physiological solutions. ${ }^{[29]}$ Parallel to this, and for metallic atomic and molecular ions in solution, we identified features in the soft X-ray excitation spectra of the total X-ray fluorescence that show up as dips, rather than bands. These dips reflect a strong interaction with the aqueous solvent and are due to the fact that the X-ray fluorescence is quenched by electron transfer from the d-orbitals of the metal to the oxygen of the water molecules. The time scale is estimated to be on the order of a fs based on the core-hole lifetime. These observations are promising to what we may call attosecond radiation chemistry, as they offer an exciting subject of investigation with high harmonic generation sources of attosecond pulses. The dips are also a powerful and simple diagnostics of the interactions of the solute with the water solvent molecules. ${ }^{[30]}$ When transposed into the time domain, which is one of our aims within MUST, these features will turn out to be extremely useful to probe solvent changes, along with the ultrafast XANES and EXAFS in the hard X-ray domain.

\section{Acknowledgements}

The results presented here were supported by the Swiss NSF via grants 20002012723 , 200021-116394 and 206021-117401, the 
NCCR MUST, and by the SER via the COST actions D35 (C06.0016) and COST CM0702 (CM0702).

Received: March 1, 2011

[1] A. H. Zewail, Angew. Chem. Int. Ed. 2000, 39, 2587.

[2] a) A. H. Zewail, Annu. Rev. Phys. Chem. 2006 , 57, 65; b) C. V. Schmising, M. Bargheer, M. Woerner, T. Elsaesser, Z. Kristallogr. 2008, 223 283; c) T. K. Kim, J. H. Lee, M. Wulff, Q. Y Kong, H. Ihee, Chem. Phys. Chem. 2009, 10 1958; d) M. Chergui, A. H. Zewail, Chem. Phys. Chem. 2009, 10, 28; e) L. X. Chen, X. Y. Zhang, J. V. Lockard, A. B. Stickrath, K. Attenkofer, G. Jennings, D. J. Liu, Acta. Crystallogr. A 2010 66, 240.

[3] a) C. Bressler, M. Chergui, Chem. Rev. 2004, 104, 1781; b) C. Bressler, M. Chergui, Ann Rev. Phys. Chem. 2010, 61, 263; c) M. Chergui, Acta. Crystallogr. A 2010, 66, 229.

[4] C. Bressler, C. Milne, V. T. Pham, A. ElNahhas, R. M. van der Veen, W. Gawelda, S. Johnson, P. Beaud, D. Grolimund, M. Kaiser, C. N. Borca, G. Ingold, R. Abela, M. Chergui, Science 2009, $323,489$.

[5] P. Beaud, S. L. Johnson, A. Streun, R. Abela D. Abramsohn, D. Grolimund, F. Krasniqi, T. Schmidt, V. Schlott, G. Ingold, Phys. Rev. Lett. 2007, 99, 174801

[6] a) M. Saes, C. Bressler, F. van Mourik, W Gawelda, M. Kaiser, M. Chergui, C. Bressler, D. Grolimund, R. Abela, T. E. Glover, P. A Heimann, R. W. Schoenlein, S. L. Johnson, A. M. Lindenberg, R. W. Falcone, Rev. Sci. Instrum. 2004, 75, 24; b) W. Gawelda, C. Bressler, M. Saes, M. Kaiser, A. Tarnovsky, D. Grolimund, S. L. Johnson, R. Abela, M Chergui, Physica Scripta 2005, T115, 102; c) C. Bressler, R. Abela, M. Chergui, Z. Kristallogr 2008, 223, 307; d) F. Lima, C. J. Milne, C. V. D. Amarasinghe, R. M. van der Veen, M. Reinhard, M. H. Rittmann-Frank, D. Grolimund, C. Borca, R. Abela, F. van Mourik, M. Chergui, Rev. Sci. Instrum. 2011, submitted.
[7] a) M. Saes, C. Bressler, R. Abela, D. Grolimund, S. L. Johnson, P. A. Heimann, M. Chergui, Phys. Rev. Lett. 2003, 90, 047403; b) W. Gawelda, M. Johnson, F. M. F. de Groot, R. Abela, C. Bressler, M. Chergui, J. Am. Chem. Soc. 2006, 128, 5001.

[8] A. Cannizzo, F. van Mourik, W. Gawelda, G. Zgrablic, C. Bressler, M. Chergui, Angew. Chem. Int. Ed. 2006, 45, 3174.

[9] a) K. Nozaki, K. Takamori, Y. Nakatsugawa, T. Ohno, Inorg. Chem. 2006, 45, 6161; b) F. Alary, J. L. Heully, L. Bijeire, P. Vicendo, Inorg. Chem. 2007, 46, 3154.

[10] R. M. van der Veen, A. Cannizzo, F. van Mourik, A. Vlcek, M. Chergui, J. Am. Chem. Soc. 2011, $133,305$.

[11] R. M. van der Veen, C. J. Milne, A. El Nahhas, F. A. Lima, V. T. Pham, J. Best, J. A. Weinstein, C. N. Borca, R. Abela, C. Bressler, M. Chergui, Angew. Chem. Int. Ed. 2009, 48, 2711.

[12] R. M. van der Veen, J. J. Kas, C. J. Milne, V. T. Pham, A. El Nahhas, F. A. Lima, D. A. Vithanage, J. J. Rehr, R. Abela, M. Chergui, Phys. Chem. Chem. Phys. 2010, 12, 5551

[13] I. V. Novozhilova, A. V. Volkov, P. Coppens, J. Am. Chem. Soc. 2003, 125, 1079.

[14] A. Hauser, Top. Curr. Chem. 2004, 234, 155

[15] A. Cannizzo, C. J. Milne, C. Consani, W. Gawelda, C. Bressler, F. van Mourik, M. Chergui, Coord. Chem. Rev. 2010, 254, 2677.

[16] B. Ordejon, C. de Graaf, C. Sousa, J. Am. Chem. Soc. 2008, 130, 13961.

[17] P. Guionneau, M. Marchivie, G. Bravic, J. F. Letard, D. Chasseau, Topics in Current Chemistry 2004, 234, 97.

[18] W. Gawelda, V. T. Pham, M. Benfatto, Y. Zaushytsin, M. Kaiser, D. Grolimund, S. Johnson, R. Abela, C. Bressler, M. Chergui, Phys. Rev. Lett. 2007, 98, 057401.

[19] W. Gawelda, V. T. Pham, R. M. van der Veen, D. Grolimund, R. Abela, M. Chergui, C. Bressler, J. Chem. Phys. 2009, 130, 124520.

[20] W. Gawelda, A. Cannizzo, V. T. Pham, F. van Mourik, C. Bressler, M. Chergui, J. Am. Chem. Soc. 2007, 129, 8199.

[21] C. Consani, M. Premont-Schwarz, A. ElNahhas, C. Bressler, F. van Mourik, A.
Cannizzo, M. Chergui, Angew. Chem. Int. Ed. 2009, 48, 7184.

[22] V. T. Pham, I. Tavernelli, C. J. Milne, R. M. van der Veen, P. D’Angelo, C. Bressler, M. Chergui, Chem. Phys. 2010, 371, 24.

[23] a) S. Koneshan, J. C. Rasaiah, R. M. LyndenBell, S. H. Lee, J. Phys. Chem. B 1998, 102, 4193; b) R. M. LyndenBell, J. C. Rasaiah, J. Chem. Phys. 1997, 107, 1981.

[24] V. T. Pham, W. Gawelda, Y. Zaushytsin, M Kaiser, D. Grolimund, S. Johnson, R. Abela, C. Bressler, M. Chergui, J. Am. Chem. Soc. 2007 129, 1530.

[25] V. T. Pham, T. Penfold, C. J. Milne, R. M. van der Veen, A. El Nahhas, F. Lima, S. Johnson, R. Abela, I. Tavernelli, C. Bressler, M. Chergui, J. Am. Chem. Soc. 2011, submitted.

[26] B. Winter, M. Faubel, Chem. Rev. 2006, 106, 1176.

[27] a) N. Huse, H. D. Wen, D. Nordlund, E. Szilagyi, D. Daranciang, T. A. Miller, A. Nilsson, R. W. Schoenlein, A. M. Lindenberg, Phys. Chem. Chem. Phys. 2009, 11, 3951; b) G. Gavrila, K. Godehusen, C. Weniger, E. T. J. Nibbering, T. Elsaesser, W. Eberhardt, P. Wernet, Appl. Phys. A. Mater. 2009, 96, 11

[28] N. Huse, T. K. Kim, L. Jamula, J. K. McCusker, F. M. F. de Groot, R. W. Schoenlein, J. Am. Chem. Soc. 2010, 132, 6809.

[29] a) E. F. Aziz, N. Ottosson, S. Bonhommeau, N. Bergmann, W. Eberhardt, M. Chergui, Phys. Rev. Lett. 2009, 102, 68103; b) N. Bergmann, S. Bonhommeau, K. M. Lange, S. M. Greil, S. Eisebitt, F. de Groot, M. Chergui, E. F. Aziz, Phys. Chem. Chem. Phys. 2010, 12, 4827.

[30] E. F. Aziz, M. H. Rittmann-Frank, K. M. Lange, S. Bonhommeau, M. Chergui, Nat. Chem. 2010 , 2,853 . 